\title{
An open-label, randomized prospective study to evaluate the efficacy and safety of Carica papaya leaf extract for thrombocytopenia associated with dengue fever in pediatric subjects
}

This article was published in the following Dove Medical Press journal: Pediatric Health, Medicine and Therapeutics

\section{BK Srikanth' \\ Lakshminarayana Reddy' \\ Sunita Biradar' \\ Manjula Shamanna ${ }^{2}$ \\ Deepak D Mariguddi ${ }^{2}$ \\ M Krishnakumar ${ }^{2}$ \\ 'Department of Paediatrics, Vijayanagara Institute of Medical Sciences, Bellary, Karnataka, India; ${ }^{2}$ Department of Medical Services, Micro Labs Ltd, Bengaluru, Karnataka, India}

Correspondence: BK Srikanth Department of Paediatrics, Vijayanagara Institute of Medical Sciences, Cantonment Ballari, Bellary, Karnataka 583 I04, India Tel+9l 9900066996

Email sbk9294@gmail.com
Objective: Thrombocytopenia in dengue fever (DF) is a well-known complication in both adults and pediatric subjects. Management of DF primarily includes symptomatic and intensive supportive care. There are studies available on the efficacy and safety of Carica papaya leaf extract (CPLE) in adult patients with DF. However, there are no published studies available on the efficacy and safety of CPLE in the pediatric age group. Hence, this study was conducted.

Methodology: A prospective, open-label, randomized controlled study was conducted in subjects aged between 1 and 12 years having thrombocytopenia associated with DF (NS-1 antigen positive) or dengue hemorrhagic fever (DHF) grades I and II. All participants were randomized into the intervention group ( $n=147$, CPLE syrup + standard therapy) and the control group $(n=147$, received only standard therapy). All subjects were followed up daily for 5 days with monitoring of blood counts.

Results: A total of 285 subjects were finally evaluated for efficacy, and nine dropped out (seven in the control group and two in the intervention group). However, all 294 subjects were evaluated for safety. CPLE (Caripill) syrup increased the platelet count significantly compared to the control group $(P<0.05)$. In the intervention group, the platelet count increased from day 3 onward: platelet count on day 3 (mean platelet count $=89,739.31, P=0.030$ ), day 4 (mean platelet count $=120,788.96, P=0.019$ ), and day 5 (mean platelet count $=168,922.75 P=0.023$ ). Two children complained of nausea in the intervention group. Overall, Caripill syrup was well tolerated.

Conclusion: CPLE syrup significantly increases the platelet count in pediatric DF patients and is well tolerated.

Keywords: Caripill, viral fever, platelet, children, syrup, India

\section{Introduction}

Dengue is an acute viral infection associated with thrombocytopenia, which is of great public health concern in India. Dengue fever (DF) is a severe, flu-like illness that affects all age groups including infants, young children, and adults. DF is caused by flavivirus transmitted mainly by Aedes aegypti and also by Aedes albopictus mosquitoes. ${ }^{1}$ In India, $A$. aegypti is the main vector in most urban areas; however, $A$. albopictus is also implicated in many states. 
Characteristic manifestations of DF are continuous high fever, headache, retro-orbital pain, myalgia, arthralgia, hemorrhagic tendency in the form of petechiae or epistaxis, thrombocytopenia, and increase in hematocrit values. ${ }^{1-3}$ After the incubation period of 4-10 days, there is a rapid onset of symptoms and typically there are three phases in DF: febrile phase, critical phase, and recovery phase.

Dengue is one of the rapidly spreading mosquito-borne viral diseases worldwide. It is a major public health concern throughout the tropical and subtropical regions of the world. In the present decade, the incidence has increased in both urban and rural settings. WHO estimates that there may be 50-100 million dengue infections and half a million dengue hemorrhagic fever (DHF) worldwide every year, with an average case fatality rate of around $5 \%{ }^{4,5} \mathrm{~A}$ total of 3.6 billion people are at the risk of infection across 120 dengue-endemic countries. More than $70 \%$ of those at risk live in the Asia Pacific region, making this region an epicenter of dengue activity. ${ }^{6}$ Increasing burden of dengue has been a matter of serious concern worldwide. Dengue is widespread in India, and outbursts occur every year. The first epidemic of clinical dengue-like fever was recorded in Vellore of Tamil Nadu, and the first virologically proved epidemic of DF occurred in Kolkata and Eastern Coast of India in 1963-1964. ${ }^{1,7-9}$ Most of the cases are being reported in the monsoon and postmonsoon seasons. ${ }^{1,10}$ The first major widespread epidemics of DHF/dengue shock Syndrome(DSS) occurred in India in 1996, involving areas around Lucknow and Delhi and then it spread to all over the country. ${ }^{1}$ DF diagnosis is done by detecting anti-DV IgM antibodies or by NS-1 antigen in patients' serum depending upon day of illness. ${ }^{11}$ A single tube nested PCR is used for detection and serotyping of dengue virus. ${ }^{12}$ Dengue virus isolation in tissue culture cells and its sequencing are also being carried out for research purpose. ${ }^{13}$ There is no specific treatment for dengue; early detection and intensive supportive care are the most essential aspects of management. Thrombocytopenia usually occurs in the critical phase, and if left unattended or untreated it can lead to increased morbidity and mortality. As of now, there is no approved vaccine or allopathic medicine for the definitive management of DF. Few anecdotal reports have highlighted the ayurvedic or herbal preparations for treating thrombocytopenia in patients with dengue.

\section{Focus on Carica papaya leaf extract (CPLE): a novel therapeutic option}

C. papaya is a member of the Caricaceae family and belongs to dicotyledonous, polygamous, and diploid species. ${ }^{14} C$. papaya leaves are being used in ayurvedic medicine since many years. Its use as an anti-inflammatory agent, for its wound-healing properties, ${ }^{15}$ antitumor as well as immune modulatory effects, ${ }^{16}$ and as an antioxidant ${ }^{17}$ is being studied. Safety studies for chronic, subacute, and acute toxicities were conducted on CPLE and demonstrated to be safe for human consumption based on the OECD guidelines. ${ }^{18}$ Some of the genes have shown to influence platelet production, namely, arachidonate 12-lipoxygenase (ALOX 12) and platelet-activating factor receptor (PTAFR). An upsurge in the activity of these genes is necessary for platelet production and activation. The ALOX 12 gene is intensely expressed in megakaryocytes, and it has shown to be accountable for the 12-hydroxyeicosatetraenoic acid (12-HETE) production of platelets in patients treated with CPLE extract. ${ }^{19}$ On megakaryocytes, PTAFR gene is expressed, which indicates that it might be a precursor for platelet production. Clinical evidence shows that $C$. papaya extract enhances the PTFAR activity around 13-fold and the ALOX 12 activity 15-fold, which is responsible for increased platelet production. ${ }^{20-22}$ With this established mechanism of action, in few countries the leaf extract of $C$. papaya has been used in DF disease. ${ }^{21,22}$ However, there is limited literature available regarding the therapeutic options for the management of DF-induced thrombocytopenia in the pediatric age group. Hence, this study was conducted to assess the efficacy and safety of CPLE, for thrombocytopenia associated with DF in pediatric subjects.

\section{Materials and methods}

An open-label, randomized, prospective study was conducted in Vijaynagar Institute of Medical Sciences Hospital in pediatric subjects with dengue (NS1 antigen positive) associated with thrombocytopenia or DHF grades I and II.

A total of 294 subjects were randomized into intervention and control groups of 147 each. Two were dropped out from the intervention group since they withdrew from the study and got admitted into some different hospitals. Seven were dropped out from the control group. So, a total of 285 subjects were evaluated $(n=145$ in the interventional group and $n=140$ in the control group).

Both the groups were managed with the standard management. In addition, the intervention group received CPLE (Caripill) syrup formulation according to the age groups: children aged between 1 and 5 years received $275 \mathrm{mg}$ three times daily and children above 5 years received $550 \mathrm{mg}$ three times for 5 days. The control group received standard treatment only. All the subjects were followed up for plate- 
let count, red blood cell (RBC) count, and white blood cell (WBC) count daily for 5 days.

The inclusion criteria to participate in this study are as follows. Males and females between 1 and 12 years of age, who are confirmed to have DF or DHF grades I and II by NS1 antigen test and thrombocytopenia with a platelet count of 30,000-100,000/ $\mathrm{L}$, were included in the study. Subjects with a baseline alanine transaminase level of not more than three times of the upper limit of the normal range and are willing to give informed consent to participate in the study were included in the study.

Subjects with DHF grades III and IV, platelet count less than $30,000 / \mathrm{mL}$, subjects who have received blood or blood product transfusion during the current illness, and subjects with idiopathic thrombocytopenia purpura (ITP), leukemia, and hemophilia were excluded from the study. Also, subjects who have a serum ALT level three times higher than the upper limit of the normal range ( $>165 \mathrm{U} / \mathrm{L})$ and impaired renal function with serum creatinine $>1.5 \mathrm{mg}$ / $\mathrm{dL}$ (males) and $>1.4 \mathrm{mg} / \mathrm{dL}$ (females) were excluded from the trial. Subjects who participated in another trial with an investigational drug within 1 month prior to this trial, hypersensitivity to any of the components of the formulation, and the presence of any other condition that leads the investigator to conclude that the subjects are inappropriate for inclusion in this clinical study were excluded from the study.

The primary outcome measured was a mean increase in the platelet counts from the baseline levels to the end of therapy (Day 5); secondary outcomes were change in the $\mathrm{RBC}$ and WBC counts from the baseline levels till the end of therapy (Day 5) and tolerability and safety assessment based on adverse effects as mentioned by the parents/guardians and evaluated by the investigator.

Written informed consent was obtained from their parents or guardians. Institutional ethics committee (Vijayanagara Institute of Medical Sciences, Bellary) approval was obtained before initiation of the study, and the trial has been registered in the clinical trial registry, India (CTRI registration number: CTRI/2018/03/012792). The CONSORT flow diagram is shown in Figure 1.

The platelets of both the groups were monitored every day along with RBC and WBC counts. All the subjects were monitored for any adverse events throughout the study period.

Independent $t$-test was used to analyze the mean platelet, $\mathrm{RBC}$, and WBC counts between the groups. Paired $t$-test was used to compare the mean values of the parameters in the intervention group before and after treatment (ie, baseline and at the end of 5 days). Significance levels were set at $P$-value $\leq 0.05$.

\section{Results}

This study was carried out in 285 pediatric subjects diagnosed with dengue with thrombocytopenia; of whom, 145 subjects ( 77 males and 68 females) were included in the interventional group and 140 subjects (66 males and 74 females) in the control group (Figure 1).

Mean age in the intervention group was $7.43 \pm 3.61$ and the control group was $8.07 \pm 2.92$. The demographic characteristics (mean age, gender, height, and weight) are mentioned in Table 1.

In both groups, subjects presented with symptoms of dengue, ie, high fever, headache, retro-orbital pain rash, and joint pain, and diagnosis was confirmed with NS1 antigen test. Mean increase in the platelet count was considered as the primary outcome, and mean increase in $\mathrm{RBC}$ and $\mathrm{WBC}$ counts was considered as the secondary outcome.

Results indicate that there is consistent increase in the platelet count in the intervention group and the control group. In the intervention group, there is statistically significant increase compared to the control group. The increase in the platelet count in the intervention group was statistically significant on Day 3 (mean platelet count $=89,739.31 \pm 29,973.62, P=0.030)$, Day 4 (mean platelet count $=120,788.96 \pm 36,404.03, P=0.019)$, and Day 5 (mean platelet count $=168,922.75 \pm 49,706.55 P=0.023$; Figure 2).

As a part of secondary outcome assessment, mean increase in RBC count and WBC count was analyzed in both intervention and control groups. It has been observed in the study that there is an increase in the RBC and WBC counts in both groups; however, the mean increase was statistically not significant in the control group. In the intervention group, it has been observed that the mean increase in RBC count was found to be statistically significant on Day 5 (mean RBC count $=3.89 \pm 0.45 \times 10^{6} / \mu \mathrm{L}, P=0.03$ ), and the mean increase in WBC count was statistically significant on Day 3 (mean WBC count $=4.74 \pm 0.46 \times 10^{3} / \mu \mathrm{L}, P=0.038$ ), Day 4 (mean WBC count $=5.42 \pm 0.56 \times 10^{3} / \mu \mathrm{L}, P=0.047$ ), and Day 5 (mean WBC count $\left.=6.65 \pm 0.65 \times 10^{3} / \mu \mathrm{L}, P=0.008\right)$. The graphs of mean change in the RBC count and WBC count are shown in Figures 3 and 4, respectively.

All 294 subjects were evaluated for safety and tolerability. Two subjects complained of nausea in the intervention group. Overall, Caripill syrup was well tolerated in all other subjects. 


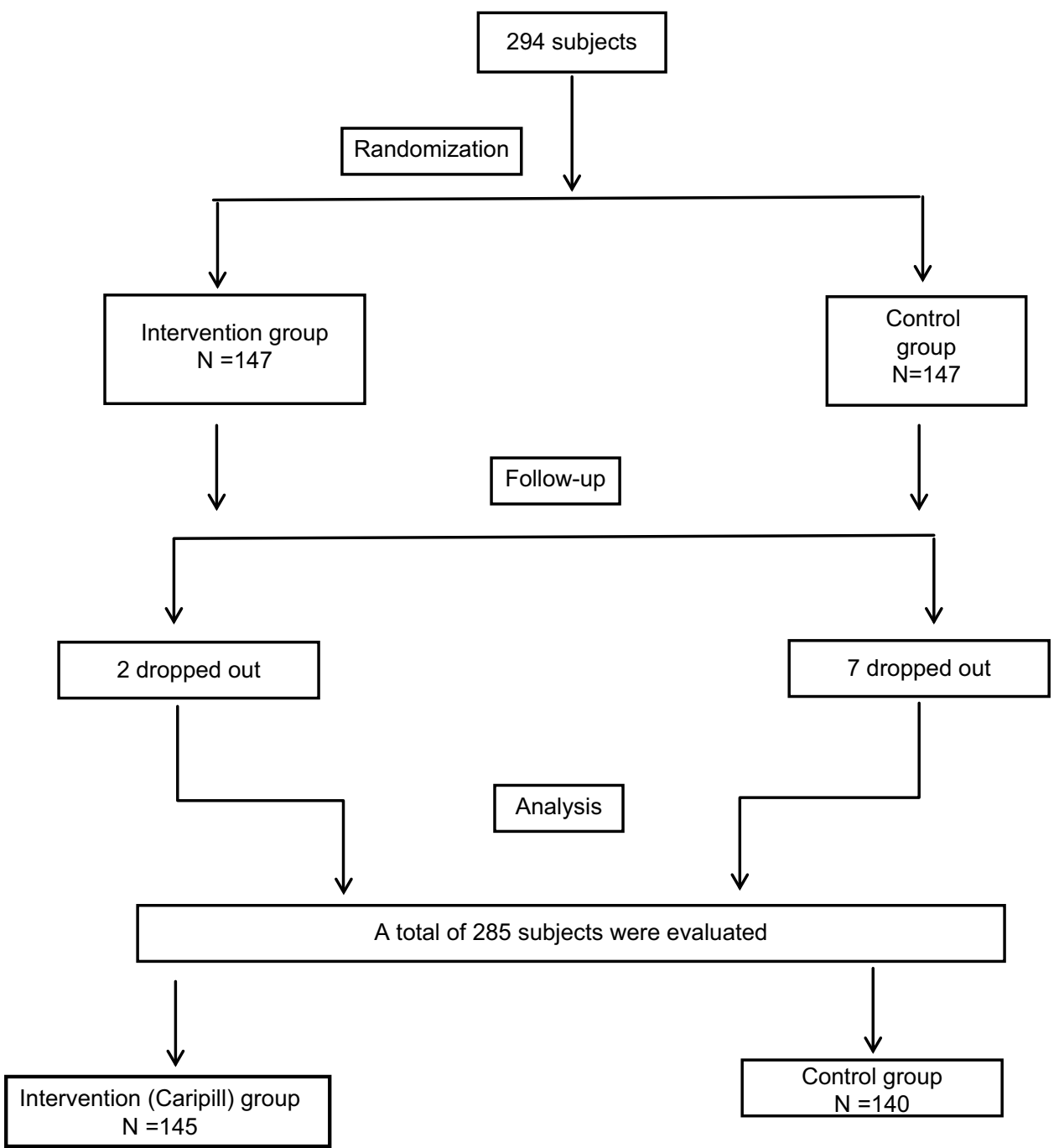

Figure I CONSORT flow diagram.

Table I Demographic characteristics

\begin{tabular}{|l|l|l|}
\hline & $\begin{array}{l}\text { Intervention group } \\
(\mathbf{n}=145)\end{array}$ & $\begin{array}{l}\text { Control group } \\
(\mathbf{n}=140)\end{array}$ \\
\hline $\begin{array}{l}\text { Age in years } \\
\text { Gender }\end{array}$ & $7.43 \pm 3.6 \mathrm{I}$ & $8.07 \pm 2.92$ \\
$\quad$ Male, $\mathrm{n}(\%)$ & $77(53.1)$ & $66(47.14)$ \\
Female, $\mathrm{n}(\%)$ & $68(46.89)$ & $74(52.85)$ \\
Height in cm & $121.16 \pm 37.92$ & $126.82 \pm 28.23$ \\
Weight in kg & $23.71 \pm 7.12$ & $25.2 \pm 5.86$ \\
\hline
\end{tabular}

Note: Data shown as mean \pm SD or $n$ (\%).

\section{Discussion}

Thrombocytopenia is a well-known complication of the $\mathrm{DF}^{23}$

Thrombocytopenia is often characterized by bruising, purpura in forearms, pinpoint hemorrhages, epistaxis, and bleeding gums. Important causes of thrombocytopenia in DF are bone marrow suppression in early stage, disseminated intravascular coagulation, peripheral sequestration of platelets, destruction of platelets (antiplatelet antibodies). Severe thrombocytopenia is associated with complications such as DHF and DSS, and if untreated leads to morbidity and mortality. ${ }^{23,24}$

DF affects all the age groups and especially the pediatric age group is at high risk. ${ }^{25}$ Infants with DF typically have high fever that usually lasts 2-7 days as seen in older children. However, compared to older children, gastrointestinal symptoms such as vomiting, diarrhea, respiratory tract symptoms (nasal congestion, runny nose, cough, and dyspnea), and febrile convulsions are more common in infants with dengue. ${ }^{25}$

Clinical features and laboratory findings of infants infected with dengue become more prominent during this critical phase. Petechiae, mucosal membrane bleeding (eg, nose and gums), and gastrointestinal bleeding may occur. Leukopenia and thrombocytopenia are often observed in this phase. Progression of the disease course of dengue in 


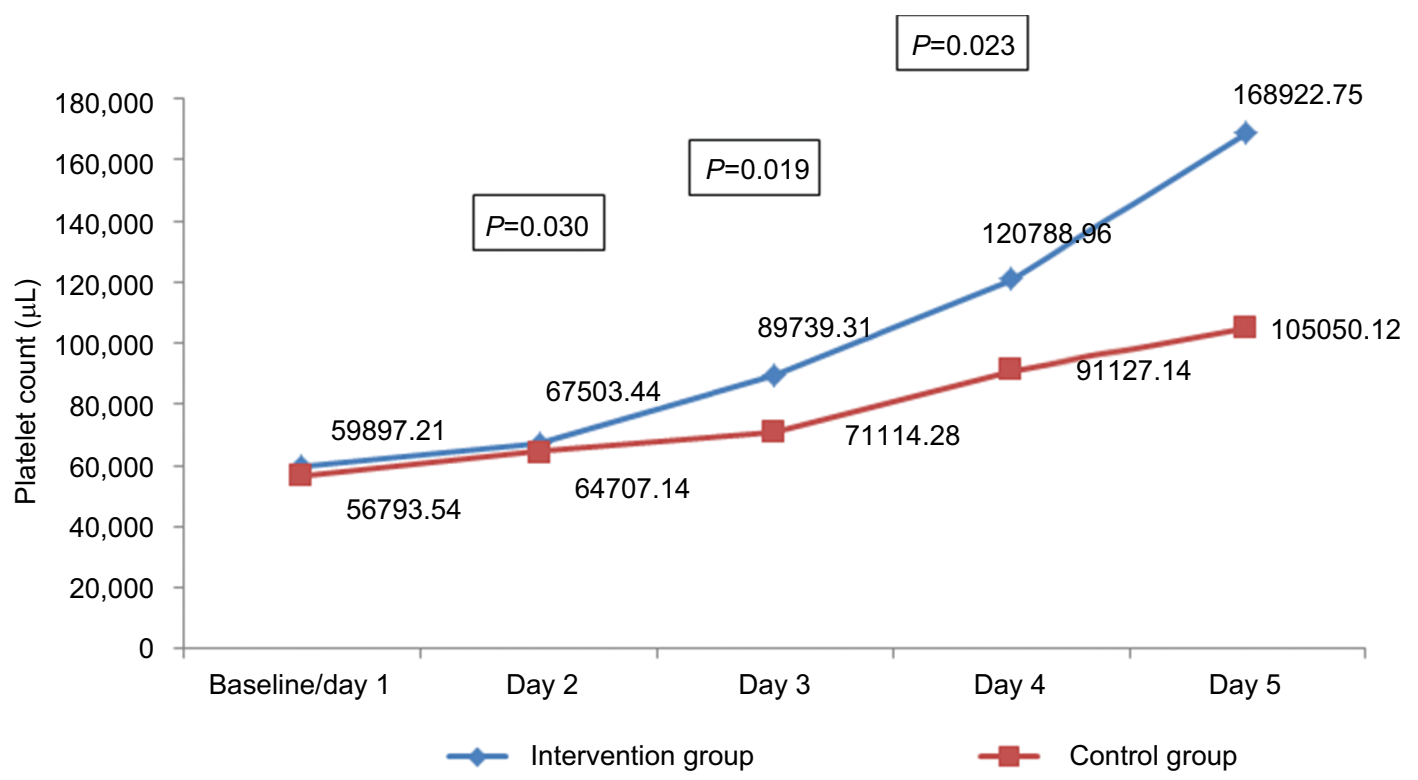

Figure 2 Changes in the platelet count in the study subjects.

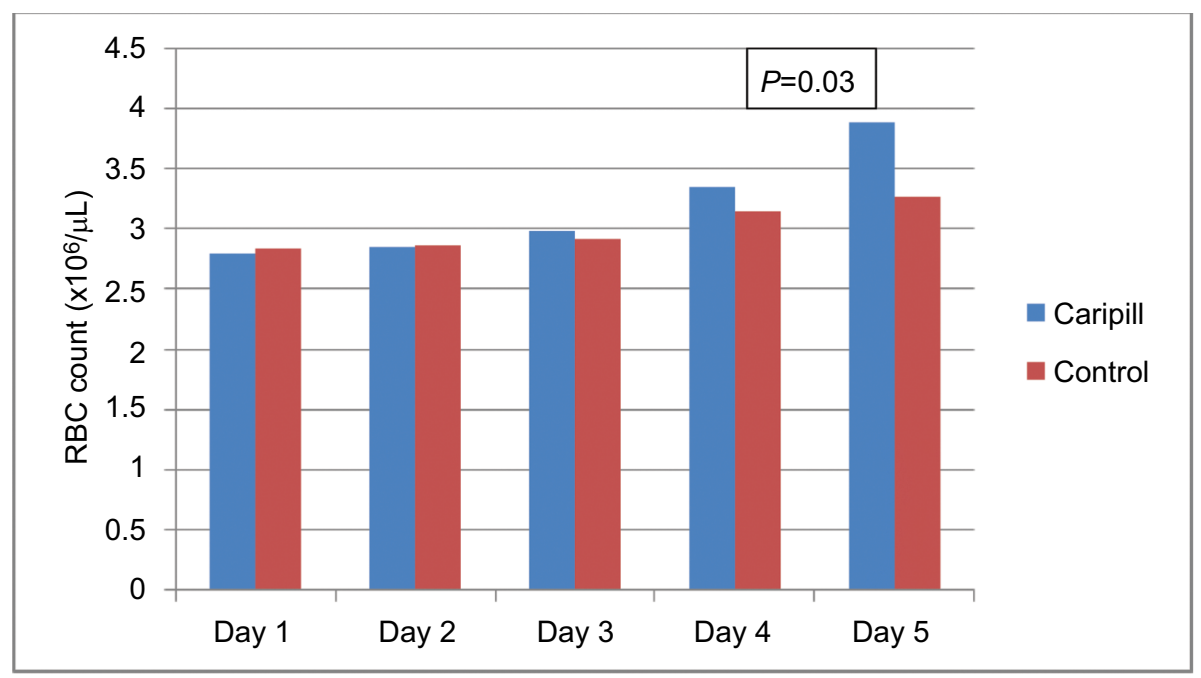

Figure 3 Mean change in RBC count.

Abbreviation: RBC, red blood cell.

infants is the same as that of children during the recovery phase. $^{25}$

This study is one of the first studies to evaluate the CPLE in pediatric patients with DF. Studies conducted in adult patients with dengue have shown that CPLE had significant increase $(P<0.01)$ in the platelet count over the therapy duration, in DF patients, confirming that CPLE accelerates the increase in platelet count compared to the control group. ${ }^{26}$
Furthermore, study conducted by many active components of C. papaya extract inhibits immune-mediated platelet destruction, bone marrow suppression, and stabilizes the membrane of infected cells in dengue. Papaya leaf extract raised the platelet count by Day 3 with decreased requirement of platelet transfusion. The drug did not produce any major side effects. ${ }^{27}$

In this study, similar results were observed in pediatric patients with dengue. CPLE (Caripill) syrup increased 


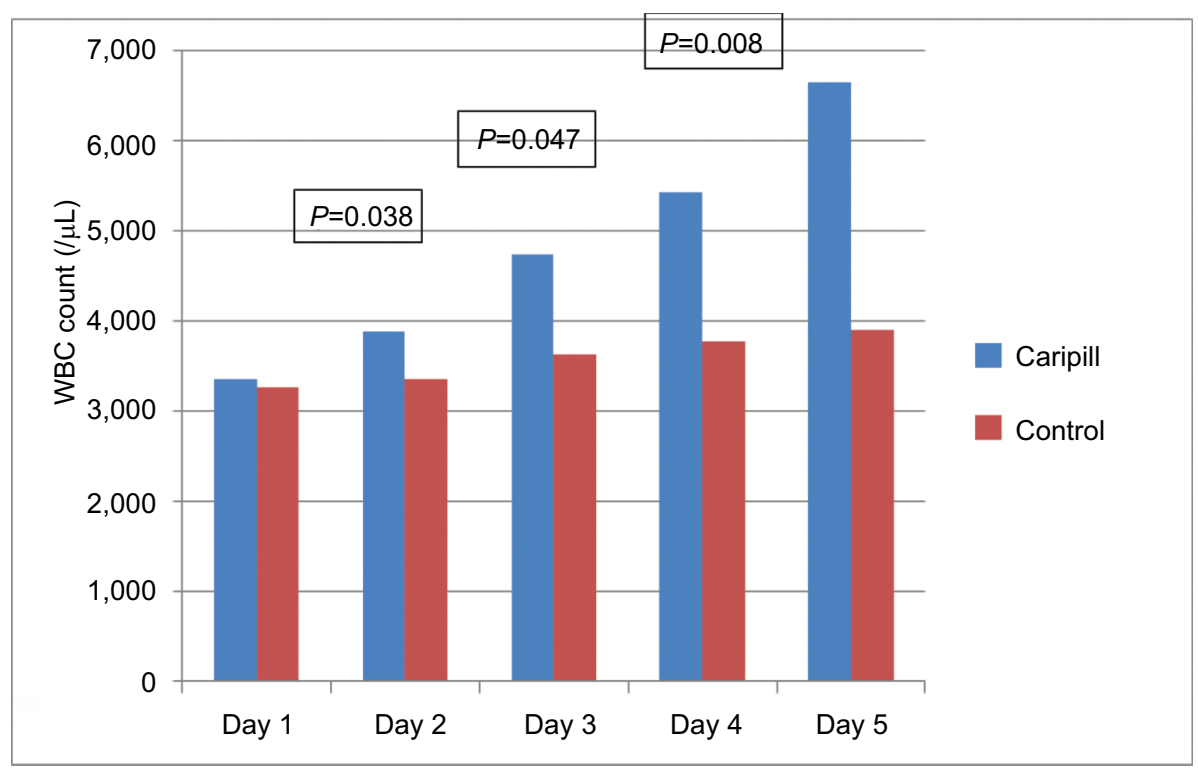

Figure 4 Mean change in WBC count.

Abbreviation: WBC, white blood cell.

the platelet count significantly compared to the control group $(P<0.05)$. In the intervention group, platelet count increased from Day 3 onward: platelet count on Day 3 (mean platelet count $=89,739.31, P=0.030$ ), Day 4 (mean platelet count $=120,788.96, P=0.019$ ), and Day 5 (mean platelet count=168,922.75 $P=0.023$ ).

Caripill syrup may have positive impact on RBC and WBC counts in pediatric subjects with DF based on the study results. Caripill syrup was well tolerated by all the patients. No major adverse events were reported.

\section{Conclusion}

In this open-label, randomized prospective study, CPLE (Caripill) syrup is shown to increase the mean platelet count in thrombocytopenia associated with DF in pediatric subjects. The syrup was well tolerated, and it can add to the therapeutic armamentarium in the management of DF as supportive therapy.

\section{Acknowledgment}

The Caripill samples for the clinical trial were provided by Micro Labs Ltd.

\section{Disclosure}

Manjula Shamanna, Deepak D Mariguddi, and M Krishnakumar are employees of Micro Labs Ltd, the makers of
Caripill (Carica papaya leaf extract) syrup. The authors report no other conflicts of interest in this work.

\section{References}

1. Gupta N, Srivastava S, Jain A, Umesh C. Chaturvedi Indian Council of Medical Research, New Delhi \& Department of Microbiology K G Medical University, Lucknow, India, Centenary Review Article. Indian J Med Res. 2012;136:373-390.

2. Gubler DJ, Mahy BWJ, Van Regenmortel MHV. Desk Encyclopedia of Human and Medical Virology. Boston: Academic Press; 2010:372-382.

3. Ranjit S, Kissoon N. Dengue hemorrhagic fever and shock syndromes. Pediatr Crit Care Med. 2011;12(1):90-100.

4. WHO. Dengue: Guidelines for Diagnosis, Treatment, Prevention, and Control in Sub-Saharan Africa and 13 Countries in South America. Geneva: World Health Organization; 2009.

5. WHO. Dengue and Dengue Haemorrhagic Fever. Factsheet No 117, Revised May 2008. Available from http://www.who.int/mediacentre/ factsheets/fs117/en/. Accessed November 10, 2018.

6. Ng LC. Challenges in dengue surveillance and control. West Pac Surveill Response J. 2011;2(2):1-3.

7. Sarkar JK, Chatterjee SN, Chakravarty SK. Haemorrhagic 5. fever in Calcutta: some epidemiological observations. Indian J Med Res. 1964;52:651-659.

8. Chatterjee SN, Chakravarti SK, Mitra AC, Sarkar JK. Virological investigation of cases with neurological complications during the outbreak of haemorrhagic fever in Calcutta. J Indian Med Assoc. 1965;45(6):314-316.

9. Carey DE, Myers RM, Reuben R, Rodrigues FM. Studies on dengue in Vellore, South India. Am J Trop Med Hyg. 1966;15(4):580-587.

10. Garg P, Nagpal J, Khairnar P, Seneviratne SL. Economic burden of dengue infections in India. Trans R Soc Trop Med Hyg. 2008;102(6): 570-577.

11. Chakravarti A, Kumar A, Malik S. Detection of dengue infection by combining the use of an NS1 antigen based assay with antibody detection. Southeast Asian J Trop Med Public Health. 2011;42(2):297-302. 
12. Mishra B, Sharma M, Pujhari SK, et al. Utility of multiplex reverse transcriptase-polymerase chain reaction for diagnosis and serotypic characterization of dengue and chikungunya viruses in clinical samples. Diagn Microbiol Infect Dis. 2011;71(2):118-125.

13. Kadhiravan T, Saxena A, Singh A, Broor S, Sharma SK, Mitra DK. Association of Intracellular T(H)1-T(H)2 Balance in CD4+ T-cells and MIP- $1 \alpha$ in CD8+ T-cells with Disease Severity in Adults with Dengue. Immune Netw. 2010;10(5):164-172.

14. Arumuganathan K, Earle ED. Nuclear DNA content of some important plant species. Plant Mol Biol Report. 1991;9(3):208-218.

15. Gurung S, Skalko-Basnet N. Wound healing properties of Carica papaya latex: in vivo evaluation in mice burn model. J Ethnopharmacol. 2009;121(2):338-341.

16. Otsuki N, Dang NH, Kumagai E, Kondo A, Iwata S, Morimoto C. Aqueous extract of Carica papaya leaves exhibits anti-tumor activity and immunomodulatory effects. J Ethnopharmacol. 2010;127(3):760-767.

17. Imaga NA, Gbenle GO, Okochi VI. Phytochemical and antioxidant nutrient constituents of Carica papaya and parquetina nigrescens extracts. Sci Res Essays. 2010;5(16):2201-2205.

18. Halim SZ, Abdullah NR, Afzan Z, Abdul Rashid BA, Jantan I, Ismail Z. Acute toxicity of Carica papaya leaf extract in Sprague Dawley rats. J Med Plants Res. 2011;5(10):1867-1872.

19. Kaur G, Jalagadugula G, Mao G, Rao AK. RUNX1/core binding factor A2 regulates platelet 12-lipoxygenase gene (ALOX12): studies in human RUNX1 haplodeficiency. Blood. 2010;115(15):3128-3135.

20. Dharmarathna SL, Wickramasinghe S, Waduge RN, Rajapakse RP, Kularatne SA. Does Carica papaya leaf-extract increase the platelet count? An experimental study in a murine model. Asian Pac J Trop Biomed. 2013;3(9):720-724.
21. Ahmad N, Fazal H, Ayaz M, Abbasi BH, Mohammad I, Fazal L. Dengue fever treatment with Carica papaya leaves extracts. Asian Pac J Trop Biomed. 2011;1(4):330-333.

22. Subenthiran S, Choon TC, Cheong KC, et al. Carica papaya leaves juice significantly accelerates the rate of increase in platelet count among patients with dengue fever and dengue haemorrhagic fever. Evid Based Complement Alternat Med. 2013;2013:616-737.

23. Esler D. Dengue - Clinical and public health ramifications. Aust Fam Physician. 2009;38(11):876-879.

24. Petres C.J. Infections caused by arthropod and rodent borne viruses. In: Longo D.L., Fauci A.S., Kasper D.L., Hauser S.L., Jameson, Halstead S.B. Etiologies of the experimental dengues of Siler and Simmons. Am J Trop Med Hygiene. 1974;23:974-982.

25. WHO. National Guidelines for clinical management of dengue fever; National vector borne disease control program. 2014. Available from: http://www.searo.who.int/india/publications/national_guidelines_clinical_management_dengue1.pdf?ua=1. Accessed November 10, 2018.

26. Kasture PN, Nagabhushan KH, Kumar A. A multi-centric KA, double-blind P-controlled. A multi-centric, double-blind, placebocontrolled, randomized, prospective study to evaluate the efficacy and safety of carica papaya leaf extract, as empirical therapy for thrombocytopenia associated with dengue fever. JAssoc Physicians India. 2016;64(6):15-20.

27. Gadhwal AK, Ankit BS, Chahar C, Tantia P, Sirohi P, Agrawal RP. Effect of carica papaya leaf extract capsule on platelet count in patients of dengue fever with thrombocytopenia. J Assoc Physicians India. 2016;64(6):22-26
Pediatric Health, Medicine and Therapeutics

\section{Publish your work in this journal}

Pediatric Health, Medicine and Therapeutics is an international, peerreviewed, open access journal publishing original research, reports, editorials, reviews and commentaries. All aspects of health maintenance, preventative measures and disease treatment interventions are addressed within the journal. Practitioners from all disciplines are invited to submit

\section{Dovepress}

their work as well as healthcare researchers and patient support groups. The manuscript management system is completely online and includes a very quick and fair peer-review system. Visit http://www.dovepress.com/ testimonials.php to read real quotes from published authors. 